\title{
Hidden surface states on pristine and H-passivated Ni(111): Angle-resolved photoemission and density-functional calculations
}

\author{
Jorge Lobo-Checa, ${ }^{1,2, *}$ Taichi Okuda, ${ }^{1,3}$ Matthias Hengsberger, ${ }^{1}$ Luc Patthey, ${ }^{2}$ Thomas Greber, ${ }^{1}$ \\ Peter Blaha, ${ }^{4}$ and Jürg Osterwalder ${ }^{1}$ \\ ${ }^{1}$ Physik-Institut, Universität Zürich, Winterthurerstrasse 190, CH-8057 Zürich, Switzerland \\ ${ }^{2}$ Swiss Light Source, Paul Scherrer Institut, CH-5232 Villigen, Switzerland \\ ${ }^{3}$ The Institute for Solid State Physics, The University of Tokyo, Kashiwa 277-8581, Japan \\ ${ }^{4}$ Technical University of Vienna, Getreidemarkt 9/165, A-1060 Vienna, Austria
}

(Received 17 July 2007; revised manuscript received 1 October 2007; published 15 February 2008)

\begin{abstract}
By means of angle-resolved photoemission, we have uncovered surface related states on the pristine and hydrogen saturated $\mathrm{Ni}(111)$ surfaces. Near normal emission spectra were recorded at room temperature as a function of photon energy. A hidden Tamm surface state is found on the clean $\mathrm{Ni}(111)$ surface at a binding energy of $\sim 1.19 \mathrm{eV}$, completely masked by the $\Lambda_{1}$ bulk $d$ band. The existence of this surface state is in agreement with the density-functional theory calculations presented here. On the other hand, a surface state related to hydrogen adsorbed on the surface has been identified at a binding energy of $\sim 0.22 \mathrm{eV}$. Under hydrogen exposure, it grows at the same rate as the three other surface states of the clean $\mathrm{Ni}(111)$ vanish.
\end{abstract}

DOI: 10.1103/PhysRevB.77.075415

PACS number(s): 73.20.At, 73.20.Hb, 79.60.Bm, 71.15.Mb

\section{INTRODUCTION}

Surface states are a consequence of a strong modification of the electronic structure in a solid induced by its surface and due to the breaking of the three-dimensional periodicity. ${ }^{1}$ Depending on the type of bulk bands they originate from, two types of surface states have been traditionally distinguished: ${ }^{2-4}$ Tamm states and Shockley states. Tamm states, initially postulated in 1932,5 are related to localized bulk states, like in $f$ and $d$ bands, and are mathematically derived using the tight-binding approximation. On the other hand, the Shockley surface states, known since $1939,{ }^{6}$ originate from delocalized states, like those in $s$ and $p$ bands, and have been theoretically derived using the nearly-free-electron approximation. In real nonideal crystal surfaces, the distinction between Shockley and Tamm surface states is sometimes blurred, and can only be differentiated by the mathematical approximation used in their derivation. ${ }^{2-4}$

On Ni(111) two different surface states near normal emission, $S_{1}$ and $S_{2}$, are known. ${ }^{7-12} S_{1}$ is the surface state closest to the Fermi energy with an electronlike (upward) dispersion (maximum binding energy $\sim 50 \mathrm{meV}$ ). $S_{2}$, on the other hand, is slightly further away from the Fermi energy and shows a holelike (downward) dispersion (minimum binding energy $\sim 250 \mathrm{meV}$ ). Recent theoretical works ${ }^{13-15}$ have shown that both surface states are spin split into two components. In the case of $S_{1}$, only the majority component (the one with the highest binding energy) shows a significant contribution to the electronic spectral weight at the $\bar{\Gamma}$ point. This component is derived mainly from $p_{z}$ states at normal emission and shows an increasing $d$ character away from it. On the other hand, it is the minority component of $S_{2}$ that shows significant weight at the surface layer in the electronic spectral weight at the $\bar{\Gamma}$ point. This component is once again mainly derived from $p_{z}$ states at normal emission and shows an increasing $d$ character away from it. The other two components of both surface states, the $S_{1}$ minority and the $S_{2}$ majority states, are, indeed, surface resonances that strongly hybridize with the underlying $d$ bulk bands; they contribute weakly to the surface spectral weight away from normal emission.

In principle, a third surface state, $S_{3}$, with a more localized Tamm character should be expected to originate from the deeper lying $d$ band of $\mathrm{Ni}(111)$ in the existing local $d$-band energy gap, with a higher binding energy than the previous two. ${ }^{16}$ This ought to be a direct consequence of the Tamm states being constituted mainly by the $d$ states of the outermost atomic layer. Even though the electronic structure of $\mathrm{Ni}(111)$ has already been studied in great detail combining angle-resolved photoemission (ARPES) with synchrotron radiation $^{7,15,17,18}$ and gas discharge lamps, ${ }^{12,19,20}$ by means of two-photon photoemission ${ }^{21-23}$ and by inverse photoemission, ${ }^{10,24}$ to our knowledge, no published work makes any reference to the existence of this surface state.

The high sensitivity of surface states to surface defects or adsorbates ${ }^{7,25,26}$ can be used to uncover hidden surface states by directly comparing the pristine and the modified surface. Hydrogen is known to adsorb dissociatively onto $\mathrm{Ni}$ surfaces, ${ }^{27,28}$ and, particularly, the surface of $\mathrm{Ni}(111)$ remains unreconstructed under $\mathrm{H}$ exposure. The presence of hydrogen on the surface is known to strongly affect the surface states of $\mathrm{Ni}(111) .{ }^{29-31}$ These are observed to shift continuously with hydrogen concentration toward higher binding energies, with a strong decrease of intensities, until they vanish at the surface saturation coverage. ${ }^{30,31}$ The hydrogen saturation coverage depends on the temperature. ${ }^{30}$ At low temperature $(80 \mathrm{~K})$, the number of hydrogen atoms that the surface can host is equal to the number of $\mathrm{Ni}$ surface atoms on $\mathrm{Ni}(111)$ [ $\sim 1 \mathrm{ML}$ (monolayer)], whereas at room temperature (RT), the coverage is reduced to half this value $(\sim 0.5 \mathrm{ML})$ according to previous studies. ${ }^{30-32}$ Theoretical work has predicted that, energetically, the most favorable adsorption site for hydrogen is at the fcc hollow site of the $\mathrm{Ni}(111)$ surface. ${ }^{27,28}$ It has been reported that the effect of hydrogen adsorption consists in a very localized (in the direction perpendicular to the surface) and uniform attractive surface potential which depends on the hydrogen concentration, ${ }^{31}$ with 
an electrostatic repulsion between the surface hydrogen atoms originating from the electronic charge transferred from the $\mathrm{Ni}$ toward the $\mathrm{H}$ atoms. ${ }^{28}$

It is the aim of this work to unravel the presence of the third surface state of $\mathrm{Ni}(111), S_{3}$. For this purpose, synchrotron radiation excited ARPES together with densityfunctional theory (DFT) calculations will be presented. Experimentally, the surface character of this state will be established in two ways: (i) by direct comparison of the clean and H-passivated experimental spectra, and (ii) by making use of the nondispersive character of the surface state energy, with the momentum perpendicular to the surface. The calculations will allow us to determine the orbital origin of this state and whether it is a pure surface state or a resonance.

\section{EXPERIMENT}

The experiments were carried out using spin-integrated ARPES in the COPHEE endstation ${ }^{33}$ located at the Surface and Interface Spectroscopy beamline at the Swiss Light Source. All measurements were performed at RT with a base pressure of the ultra-high-vacuum (UHV) vessel in the $10^{-11}$ mbar range. The data were measured mainly using linear horizontal polarized light (some data were, in addition, acquired using left elliptical polarized light) in the photon energy range from 19.5 to $85 \mathrm{eV}$. The angular resolution was better than $0.5^{\circ}$ full width at half maximum and the overall energy resolution was $30 \mathrm{meV}$ at $h \nu=30 \mathrm{eV}$.

The Ni(111) crystal was initially prepared by repeated cycles of $\mathrm{Ar}^{+}$sputtering at $0.75 \mathrm{kV}$ for 20 min followed by $18 \mathrm{~L}$ (1 Langmuir $=10^{-6}$ Torr s) of oxygen exposure at RT and subsequent flashing to approximately $750{ }^{\circ} \mathrm{C}$. The sample cleanliness was checked through the existence and quality of the known surface states, $S_{1}$ and $S_{2}$, on the slope of the $\Lambda_{3}$ bulk band at normal emission, using a photon energy of $21.2 \mathrm{eV}$. When optimum conditions were reached, x-ray photoelectron spectroscopy did not show any significant signs of contamination. From this point on, the surface could be easily refreshed by flashing to approximately $500{ }^{\circ} \mathrm{C}$ without the use of sputtering or oxygen dosing. This treatment proved to be ideal, since the pressure during flashing never rose above $3 \times 10^{-10}$ mbar. A lifetime of the clean surface of the order of $60 \mathrm{~min}$ was achieved before residual hydrogen was adsorbed on the surface in sufficient quantities to introduce changes in the ARPES data.

The binding energy of the $S_{1}$ surface state critically depends on the surface temperature, ${ }^{12}$ showing a value of $25 \mathrm{meV}$ at RT and crossing the Fermi energy at a temperature around $370 \mathrm{~K}$. In order to have stable conditions, particular care was taken to measure all the spectra at the same temperature (RT). Due to the short clean surface lifetime and in order to increase the acquisition time, we accelerated the sample cooling by means of a short flow of liquid nitrogen (2 min) after the sample flash. This allowed us to reach RT from $\sim 500{ }^{\circ} \mathrm{C}$ in approximately $15 \mathrm{~min}$.

The hydrogen passivation of the pristine $\mathrm{Ni}(111)$ surface was performed in two ways: The first one was by exposure of the RT sample to $6 \mathrm{~L}$ of $\mathrm{H}_{2}$ gas, which quenched com- pletely the $S_{1}$ and $S_{2}$ surface states. Larger hydrogen gas doses $(24 \mathrm{~L})$ did not show any further change in the photoemission spectra. The second one, leading to equivalent results as the previous method, was slower and used the spontaneous surface aging within the residual gas of the UHV vessel, which mainly consisted of $\mathrm{H}_{2}$. After $10 \mathrm{~h}$, the spectra showed no differences with those obtained using the method described first. Once the surface was passivated with hydrogen, no changes were observed in the ARPES spectra for days.

The work function of the pristine $\mathrm{Ni}(111)$ surface was found to be $5.36 \mathrm{eV}$, slightly higher than previously reported, 7,23 while for the $\mathrm{H}$-passivated surface, the value was $5.52 \mathrm{eV}$, yielding a difference of $0.16 \mathrm{eV}$ between the two surfaces, in good agreement with previous work. ${ }^{23,29}$

\section{THEORETICAL CALCULATIONS}

Self-consistent calculations of total energies and the electronic structure based on the scalar-relativistic full-potential "augmented plane wave+local orbitals" method ${ }^{34,35}$ (APW + lo) were carried out for $\mathrm{Ni}(111)$ using the WIEN2K code. ${ }^{36,37}$ This is a very accurate and efficient scheme to solve the Kohn-Sham equations of DFT, in which exchange and correlation effects are treated, for example, by the generalized gradient approximation, which often leads to better energetics and equilibrium structures than the local density approximation. ${ }^{38}$ Besides the "linearizing" local orbitals for $s, p$, and $d$ valence states of the standard APW+lo basis, additional local orbitals are added in order to describe the $3 p$ (semicore) states of $\mathrm{Ni}$. An atomic sphere radius of 2.3 a.u. was used. The required precision was achieved by using a very large plane-wave (PW) cutoff. In the linearized APW method, the relevant convergence parameter is $R K_{\max }$, which is defined as the product of the smallest atomic sphere radius times the largest reciprocal lattice vector of the PW basis. We use $R K_{\max }=10.2$. This corresponds to a PW energy cutoff of almost $20 \mathrm{Ry}$. A $k$-point sampling of up to 400 points in the full two-dimensional (2D) Brillouin zone was used. The $k$ mesh is generated in the irreducible wedge of the Brillouin zone on a special point grid which can be used in a modified tetrahedron scheme. ${ }^{39}$

The Ni(111) surface was modeled by periodic slab supercells with a vacuum region of 20 bohrs. The geometry optimization was first performed using seven Ni layers, which were found to be sufficient for this purpose. All other results were produced using this optimized geometry for the surface layers, but adding additional bulk layers, forming a thicker slab of 19 layers of Ni. Such large slabs are necessary to reduce the spurious surface-surface interactions, which, otherwise, lead to a splitting of the two known surface states. ${ }^{40}$ Preliminary calculations with a smaller vacuum region or using only seven Ni layers have shown that the two surfaces interact weakly with each other via the "bulk" as well as via the "vacuum," producing a spurious splitting of the surface states into "bonding" and "antibonding" states. This splitting vanishes by using the large slab.

The calculated majority (minority) spin band structures of the surface $\mathrm{Ni}$ atoms are shown in the left (right) graph of 

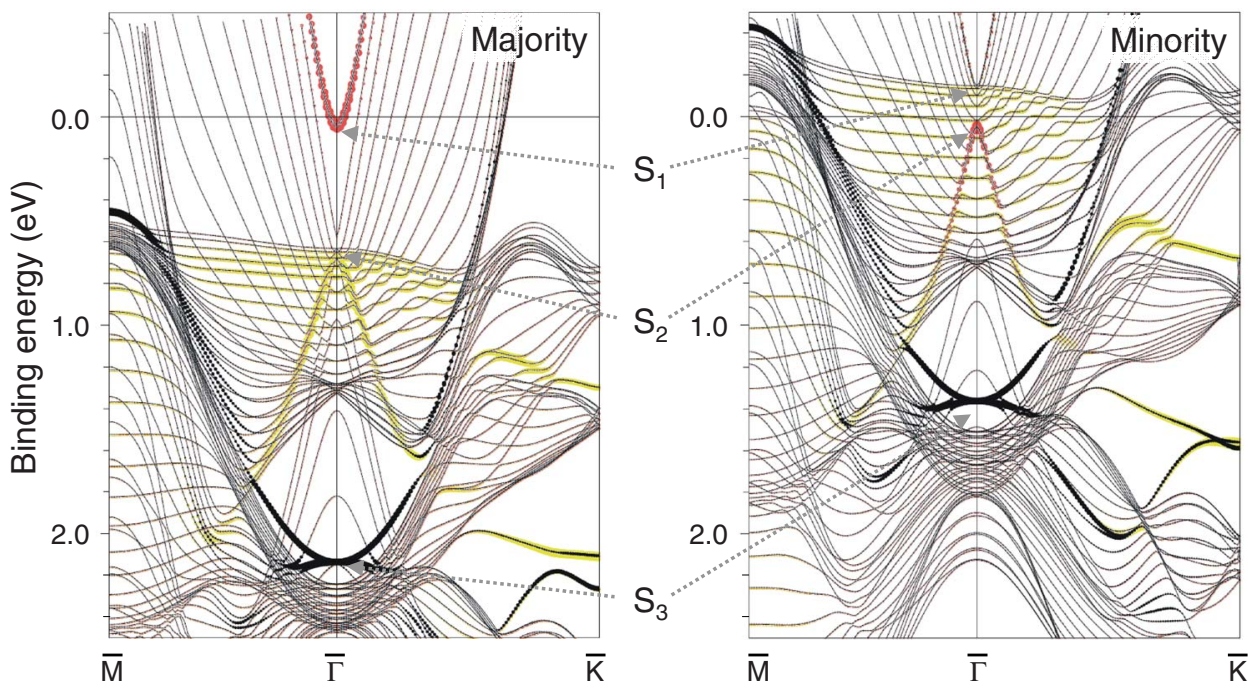

FIG. 1. (Color online) Theoretical DFT calculation of Ni(111) in the $\bar{M}-\bar{\Gamma}-\bar{K}$ directions of the 2D hexagonal reciprocal unit cell. Majority (minority) spin is represented on the left (right) graph. The thickness of the lines is proportional to the charge of the surface Ni atoms; red (dark gray), light green (light gray), and black colors indicate the $p_{z}, d_{x z, y z}$, and $d_{x y, x^{2}-y^{2}}$ characters, respectively. The existing surface states $\left(S_{1}, S_{2}\right.$, and $\left.S_{3}\right)$ have been indicated in the figure.

Fig. 1. They are in excellent agreement with other recent theoretical work. ${ }^{13}$ The different surface states at the $\bar{\Gamma}$ point are indicated by arrows in the figure: $S_{1}$ shows an electronlike (upward) dispersion, whereas $S_{2}$ has a holelike (downward) dispersion. At normal emission, an analysis of the wave function (or charge density) of the corresponding eigenvalues predicts that only the majority component of the $S_{1}$ and the minority component of $S_{2}$ surface states contribute to the electronic spectral weight. At this point, both states derive mainly from $p_{z}$ states with a $10 \%$ admixture of $d_{z 2}$ states of the surface Ni atom layer, while the contributions from subsurface atoms decrease exponentially. This orbital character progressively changes toward $d_{x z, y z}$ as the states disperse toward $\overline{\mathrm{K}}$ and $\overline{\mathrm{M}}$.

The other spin components of these states, the minority component of $S_{1}$ and the majority component of $S_{2}$, do not show the signature of a surface state at the $\bar{\Gamma}$ point, but a state with surface character reappears quickly away from this point. They are weak resonances that have such a strong hybridization with the bulk $d$ bands that they are found to be surface resonances.

The DFT calculations also predict a third surface state, which is a true surface state, with a binding energy of $1.36 \mathrm{eV}$ for the minority spin and $2.14 \mathrm{eV}$ for the majority spin, labeled $S_{3}$ in Fig. 1. It is twofold degenerate at the $\bar{\Gamma}$ point, with one branch showing upward dispersion and the other one the opposite. Even though there is only a local $d$-band gap formed between the $\Lambda_{3}$ and $\Lambda_{1}$ bulk bands, the large localization of this state on the surface atoms (about $80 \%$ ) of both spin components indicates that they are real surface states. Because of this high localization and because both states are derived from pure $d$ states, showing $d_{x y}$ and $d_{x^{2}-y^{2}}$ characters, this surface state is a Tamm state. In the following section, we will present experimental evidence for the existence of this state.
In these calculations, the spin splitting of the bulk bands $(625 \mathrm{meV})$ is clearly overestimated with respect to the experimental splitting found using spin- and angle-resolved photoemission, which was measured to be $160 \mathrm{meV}$ at RT for the $\Lambda_{3} d$-band peak in normal emission on $\mathrm{Ni}(111),{ }^{9}$ and $280 \mathrm{meV}$ for the uppermost $d$ band that crosses the Fermi level. ${ }^{19}$ In comparing the calculated binding energies to those observed in the experimental spectra, one should note that, in nickel, a severe energy renormalization with respect to such DFT calculations occurs in the spectral function-which is what the photoemission spectrum measures-due to strong electron-electron interaction in the photoemission hole state. ${ }^{41}$ Using a Green's function formalism, it was shown that the real part of the self-energy, responsible for the energy shift of the measured bands toward the Fermi energy, is strongly spin dependent, leading to a reduction of the measured exchange splitting of bulk bands by about a factor of 2 when compared to DFT calculations. This is a consequence of the localized $d$ orbitals that mix strongly with most of the bands measured in the nickel valence band, and the large Coulomb and exchange terms associated with many-body processes involving these states. So far, these effects have not been studied for the surface state spectra of nickel. For the $S_{1}$ state, the measured exchange splitting at $\bar{\Gamma}$ is $\sim 100 \mathrm{meV},{ }^{24}$ while the one predicted from the present calculations is $180 \mathrm{meV}$. A clear assignment of the $S_{2}$ state, measured at a $\bar{\Gamma}$ binding energy of $250 \mathrm{meV}$, with regards to its majority or minority character has not yet been possible. Similar arguments apply to the surface state $S_{3}$, which is a Tamm state according to its $d$-orbital weight. Nevertheless, it can be expected somewhere above the $\Lambda_{1}$ peak in the normal emission spectra.

\section{EXPERIMENTAL RESULTS AND DISCUSSION}

To experimentally confirm the Tamm surface state, $S_{3}$, predicted by the DFT calculations presented above, near nor- 
mal emission ARPES spectra will be presented in this section. In Sec. IV A, hydrogen was adsorbed in a fast way by direct exposure of the surface at RT for 1 min to $6 \mathrm{~L}$ of $\mathrm{H}_{2}$ gas, so as to compare the resulting clean and passivated spectra. In Sec. IV B, the same hydrogen coverage is reached slowly by spontaneous adsorption of the residual gas in the vessel. This will allow us to study the evolution of the electronic structure as a function of the hydrogen coverage. As indicated in the Introduction, the hydrogen saturation coverage depends on the temperature, ${ }^{30}$ but previous works ${ }^{30-32}$ indicated that the saturation coverage at RT corresponds to $\sim 0.5 \mathrm{ML}$ of hydrogen.

\section{A. Clean versus rapidly H-passivated surfaces}

In order to look for the predicted Tamm surface state, $S_{3}$, normal emission spectra at different photon energies were acquired. The gray curve in the top panel in Fig. 2 shows the photoemission spectrum of clean $\mathrm{Ni}(111)$ at $\mathrm{RT}$ for a photon energy of $35 \mathrm{eV}$ using $p$ polarized light. The two dominant peaks at binding energies of 0.54 and $1.37 \mathrm{eV}$ should correspond to the $\Lambda_{3}$ and $\Lambda_{1}$ bulk $d$ bands, respectively. On close inspection, the already known surface states ${ }^{7-12}$ can be easily observed: (i) $S_{1}$ with a binding energy of $0.025 \mathrm{eV}$ and (ii) $S_{2}$ at a binding energy of $0.28 \mathrm{eV}$. A first visual search for the theoretically expected surface state, $S_{3}$, does not seem to be successful, but we clearly observe that the $\Lambda_{1}$ peak is much broader than the $\Lambda_{3}$ bulk band. Making use of the known sensitivity of the surface states to adsorbed species, we passivated the surface with hydrogen until saturation was reached. The dotted black curve in the top panel of Fig. 2 was measured under the same conditions as the clean spectrum, except for the hydrogen saturation at the surface. There are some obvious changes in the spectrum: the $S_{1}$ and $S_{2}$ states disappear, while a new shoulder can be observed at a binding energy of $0.23 \mathrm{eV}$. Concerning the bulk states, the $\Lambda_{3}$ band remains basically unchanged, whereas the peak corresponding to $\Lambda_{1}$ experiences an ostentatious change in the peak width, apart from an apparent binding energy shift of about $0.1 \mathrm{eV}$.

The larger width and the apparent binding energy shift of the $\Lambda_{1}$ peak may be indications for several components contributing to this peak. To verify this, we performed a fit of each spectrum assuming that the two bulk bands maintain the same width and binding energy in both spectra, with or without adsorbed hydrogen. To obtain a good fit for the clean spectrum, a superposition of five Lorentzian components were necessary together with a linear background and multiplied with a Fermi-Dirac distribution. For the H-passivated spectrum, only three Lorentzian components were needed. The results of these fits are very good, and are shown in the center and bottom graphs of Fig. 2 for the clean and $\mathrm{H}$-passivated surfaces, respectively. In the clean surface spectrum, the small component near the Fermi energy corresponds to $S_{1}$, the one found at $0.28 \mathrm{eV}$ to $S_{2}$, and the last one located at $1.19 \mathrm{eV}$ must, therefore, correspond to the expected surface state $S_{3}$. In the spectrum from the $\mathrm{H}$-passivated surface, another surface state is seen at a binding energy of $0.23 \mathrm{eV}$.

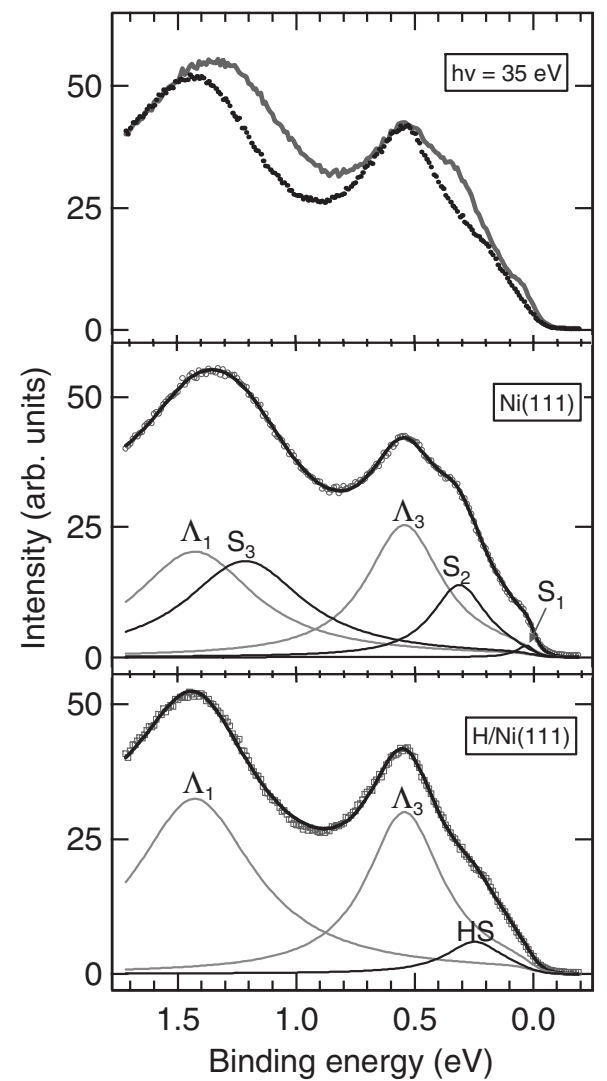

FIG. 2. Normal emission spectra measured at RT with a photon energy of $35 \mathrm{eV}$. Top panel: The thick gray line corresponds to pristine $\mathrm{Ni}(111)$ and the black dots to the $\mathrm{H}$-passivated surface. The clean and H-passivated spectra have been fitted using a linear background and a superposition of five Lorentzian components for the clean surface (center panel) and three Lorentzian components for the H-passivated surface (bottom panel), multiplied with a FermiDirac distribution (Ref. 42). The fits are shown as black thick lines and the raw data as open circles (clean) and squares (passivated). The bulk Lorentzian components are shown as thin gray lines $\left(\Lambda_{1}\right.$ and $\Lambda_{3}$ ), whereas the surface state Lorentzians are represented as thin black lines (clean Ni surface states are $S_{1}, S_{2}$, and $S_{3}$; HS corresponds to a hydrogen-induced state).

The assignment of the Lorentzian component $S_{3}$ in the fits shown in Fig. 2 as the Tamm state can be corroborated by demonstrating the nondispersive character of the surface state energy with the momentum perpendicular to the surface. At normal emission, the binding energy of the surface state should remain constant while varying the incident photon energy. For this purpose, the differences between the normal emission spectra of the clean and H-passivated surfaces were studied for a large set of photon energies, ranging from 19.5 to $85 \mathrm{eV}$. Figure 3 shows representative spectra in the photon energy range between 22 and $50 \mathrm{eV}$. For each energy, the clean spectra and the passivated spectra (shifted in intensity for a better comparison) are shown as open circles and open squares, respectively.

In the clean surface spectra, especially for low photon energies, we can clearly observe the surface states $S_{1}$ and $S_{2}$ at binding energies of 0.020 and $0.250 \mathrm{eV}$, showing no energy dispersion with the photon energy, and in excellent 

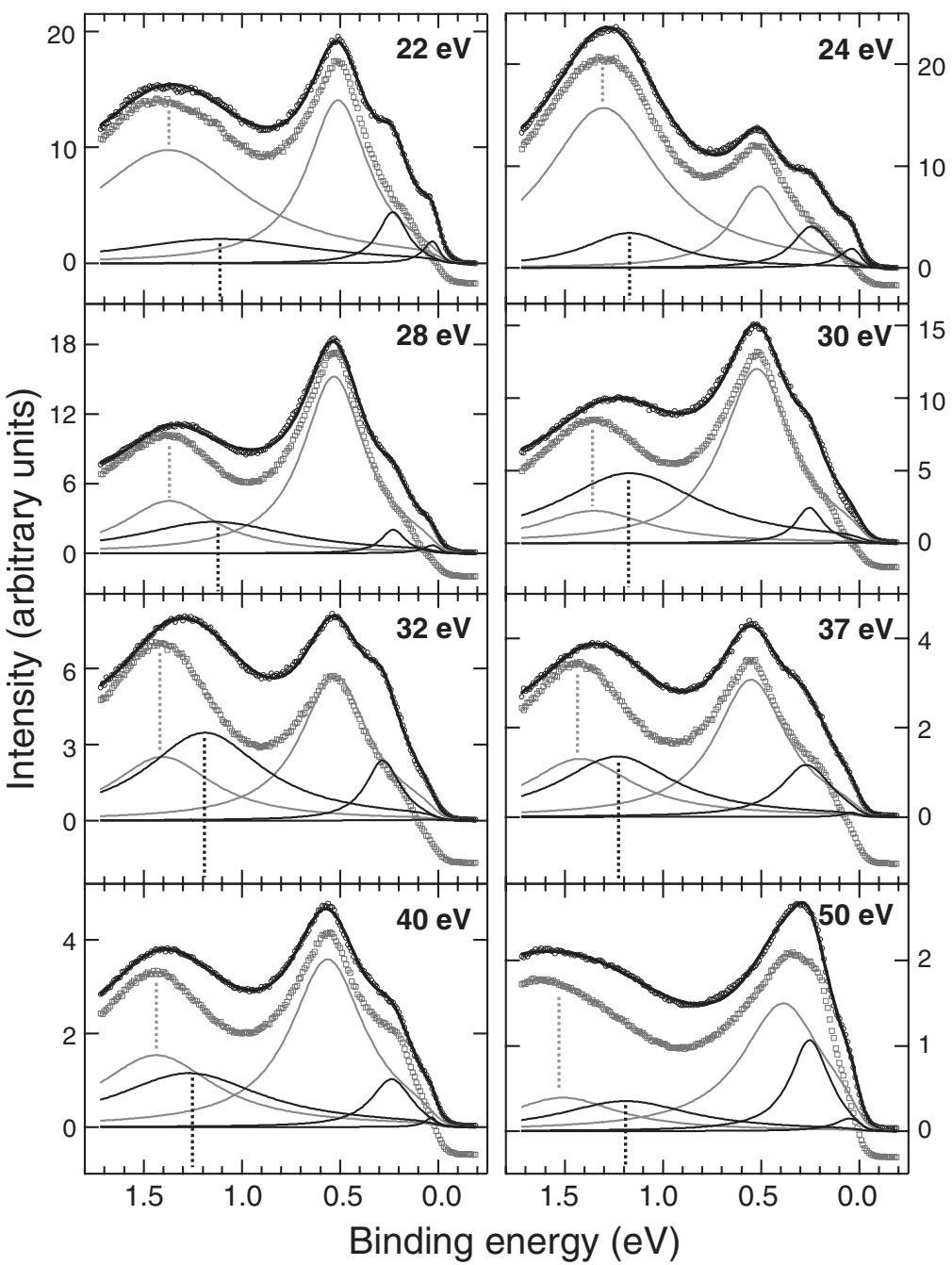

FIG. 3. Representative normal emission spectra recorded at RT for different photon energies ranging from 22 to $50 \mathrm{eV}$. The open dark-gray circles correspond to the pristine $\mathrm{Ni}(111)$, and the open light-gray squares to the H-passivated surface (shifted downward in intensity for better visualization). The fitting procedure described previously for Fig. 2 has been performed for all spectra. The resulting fit (thick black lines) and the five Lorentzian components (gray lines for the bulk components and black thin lines for the surface states) are shown for the clean spectra in each graph (Refs. 42-44). The vertical dotted black (gray) lines correspond to the energy positions of the Tamm $\left(\Lambda_{1}\right.$ bulk) components.

agreement with previous work. ${ }^{7-12}$ The two dominant peaks, which correspond to the bulk $d$ bands, show some dispersion as a function of photon energy, which is more prominent in the case of the higher binding energy peak $\left(\Lambda_{1}\right)$.

When the surface is passivated with hydrogen, the spectra undergo some important changes: The $S_{1}$ and $S_{2}$ peaks disappear and a new state appears with a binding energy of $0.22 \mathrm{eV}$, which does not disperse with photon energy. This indicates that this state is a surface state related to the hydrogen adsorption.

When comparing the two dominant peaks of the clean and passivated spectra, it can be observed that the $\Lambda_{3}$ bulk band does not change its binding energy or peak width, whereas the $\Lambda_{1}$ bulk band peak appears narrower and shifted slightly to higher binding energy in a way similar to the spectra in Fig. 2. These changes are observed to be dependent on the photon energy.
To understand the changes occurring on the $\Lambda_{1}$ peak, all the spectra measured with photon energies up to $50 \mathrm{eV}$ were analyzed with a similar procedure as the one displayed in Fig. 2. Least-square fits were carried out using a total of six Lorentzian components and a constant linear background, multiplied with a Fermi-Dirac distribution. As a first step, the spectra from the H-passivated surface were fitted with only three Lorentzians: one for each of the bulk bands and a third one for the surface state related to the hydrogen-adsorption. Then the spectra from the clean surface were fitted, assuming that the hydrogen-induced surface state is absent and that the bulk-band binding energies and widths are fixed within each photon energy, that is, only the intensities of the bulk bands were allowed to change between the H-passivated and clean surfaces.

The fits for the representative clean spectra are shown as thick black lines in Fig. 3. In every case, apart from the two 
TABLE I. Mean values of the binding energies and widths of the different surface states obtained from the fits to the normal emission spectra of pristine and $\mathrm{H}$-passivated $\mathrm{Ni}(111)$ in the photon energy range from 19.5 to $50 \mathrm{eV}$. The values are in agreement with previous published work (Refs. 7-12). The errors describe the scatter for the different photon energies.

\begin{tabular}{lcc}
\hline \hline & $\begin{array}{c}\text { Binding energy } \\
(\mathrm{eV})\end{array}$ & $\begin{array}{c}\text { Peak width } \\
(\mathrm{eV})\end{array}$ \\
\hline$S_{1}$ & $0.02 \pm 0.01$ & $0.16 \pm 0.06$ \\
$S_{2}$ & $0.25 \pm 0.03$ & $0.24 \pm 0.08$ \\
$S_{3}$ & $1.19 \pm 0.08$ & $0.8 \pm 0.2$ \\
Hydrogen SS & $0.22 \pm 0.04$ & $0.23 \pm 0.09$ \\
\hline \hline
\end{tabular}

bulk band components (shown as gray lines), three new Lorentzian components (black thin lines) were necessary to obtain proper fits of the clean surface spectra. As in Fig. 2, the low binding energy components are related to the known $S_{1}$ and $S_{2}$ states and do not disperse with the photon energy. ${ }^{43,44}$ The third component, marked by the dotted black vertical line, also shows the same behavior: it does not disperse with the photon energy. This evidences the existence of the hidden Tamm state at a binding energy of $1.19 \mathrm{eV}$. All the results for the binding energies and the peak widths of the surface states have been summarized in Table I.

With the experimental evidence for this surface state, we can easily explain the change in the appearance of the $\Lambda_{1}$ bulk band in Figs. 2 and 3: when the surface is covered with hydrogen, this surface state is quenched, resulting in an apparent change in width and a shift in binding energy of the $\Lambda_{1}$ peak. Greuter et al. ${ }^{31}$ interpreted this effect as a change in the chemical potential induced by the hydrogen atoms adsorbed on the surface. This explanation is inconsistent with our experimental results since it should produce a rigid shift of all the peaks.

The experimental dispersions of all these surface states with electron momentum parallel to the surface are shown in Fig. 4. Figures 4(a) and 4(c) correspond to the clean surface, and Fig. 4(b) to the H-passivated surface. The energy regions near the Fermi level [Figs. 4(a) and 4(b)] have been mea- sured with a photon energy of $21.2 \mathrm{eV}$, where we observe that the $\Lambda_{3}$ bulk peak is dominant and seems to disperse much less with emission angle than the surface states. In Fig. 4(a), we can easily identify the $S_{1}$ and $S_{2}$ states of the clean surface. The dispersions that we observe are in agreement with previous work: ${ }^{7-12}$ the $S_{1}$ state disperses toward the Fermi energy, forming an electron pocket, whereas the dispersion of the $S_{2}$ state is holelike (downward), moving toward the $\Lambda_{3}$ bulk band. In the case of the H-passivated surface [Fig. 4(b)], the hydrogen-induced surface state is seen as a small shoulder on the slope of the bulk $\Lambda_{3} d$ band, with binding energies located between those of the $S_{1}$ and $S_{2}$ surface states. This state disperses toward the Fermi energy like the $S_{1}$ state, but with a slightly larger effective mass $\left[m_{H S}^{*}\right.$ $=0.24 m_{0}$ and $m_{S_{1}}^{*}=0.17 m_{0}$ (Ref. 12)].

The spectra displayed in Fig. 4(c) were measured at a photon energy of $50 \mathrm{eV}$. In the displayed energy region, the dominant peak corresponds to the $\Lambda_{1}$ bulk band. Contrary to the other surface states, no significant dispersion is visible in this narrow angular range for the $S_{3}$ state. In the DFT calculations (Fig. 1), this state is predicted to be degenerate at the $\bar{\Gamma}$ point, showing both upward and downward dispersions. This behavior may be experimentally masked by the concomitant dispersion of the $\Lambda_{1}$ bulk band.

\section{B. Evolution of normal emission spectra upon residual hydrogen adsorption}

As explained in Sec. II, the hydrogen surface coverage can be slowly increased by allowing the residual hydrogen gas in the ultra-high-vacuum vessel to adsorb on the surface. Figure 5 shows the effect of residual gas adsorption on the normal emission spectra as a function of time. In order to study the evolution of the surface states as a function of hydrogen coverage, 174 spectra were consecutively measured at RT with a photon energy of $35 \mathrm{eV}$ for a total time of $8 \mathrm{~h}$ and $45 \mathrm{~min}$. The final spectrum was found to be identical to the one obtained by means of fast hydrogen adsorption.

In Fig. 5(a), selected raw spectra are shown as a function of time at time intervals of $\sim 30 \mathrm{~min}$. A fitting procedure similar to the one described previously, using six Lorentzians
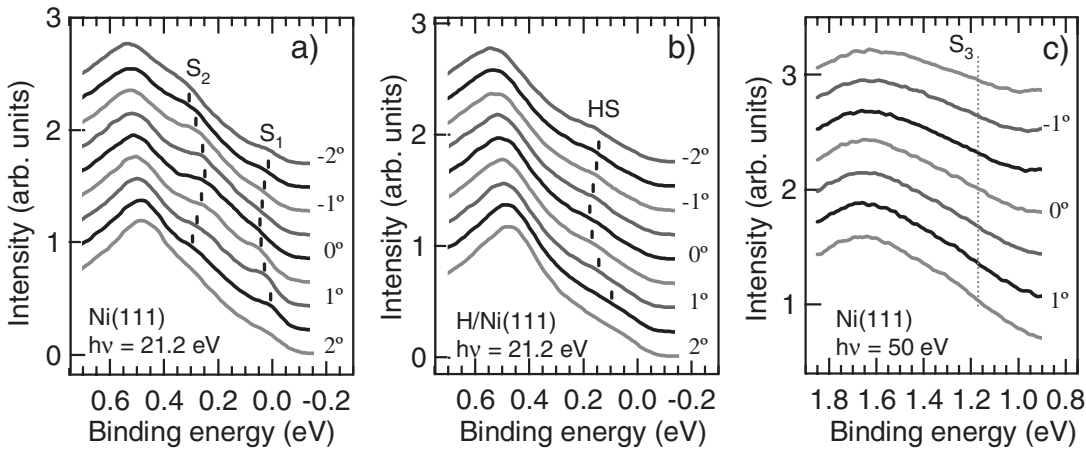

FIG. 4. Energy distribution curves for different emission angles. The angular step is $0.5^{\circ}$ and some angles are given on the right hand side of each graph. The dispersions of the different surface states are indicated by black ticks. (a) and (b) have been acquired with a photon energy of $21.2 \mathrm{eV}$, and the dispersion of the $S_{1}, S_{2}$, and hydrogen-induced surface (HS) states can be observed. In (c), no clear dispersion is observed for the Tamm state, $S_{3}$, using $50 \mathrm{eV}$ photon energy. The vertical dotted line indicates the binding energy of $S_{3}$ at normal emission $\left(0^{\circ}\right)$. 

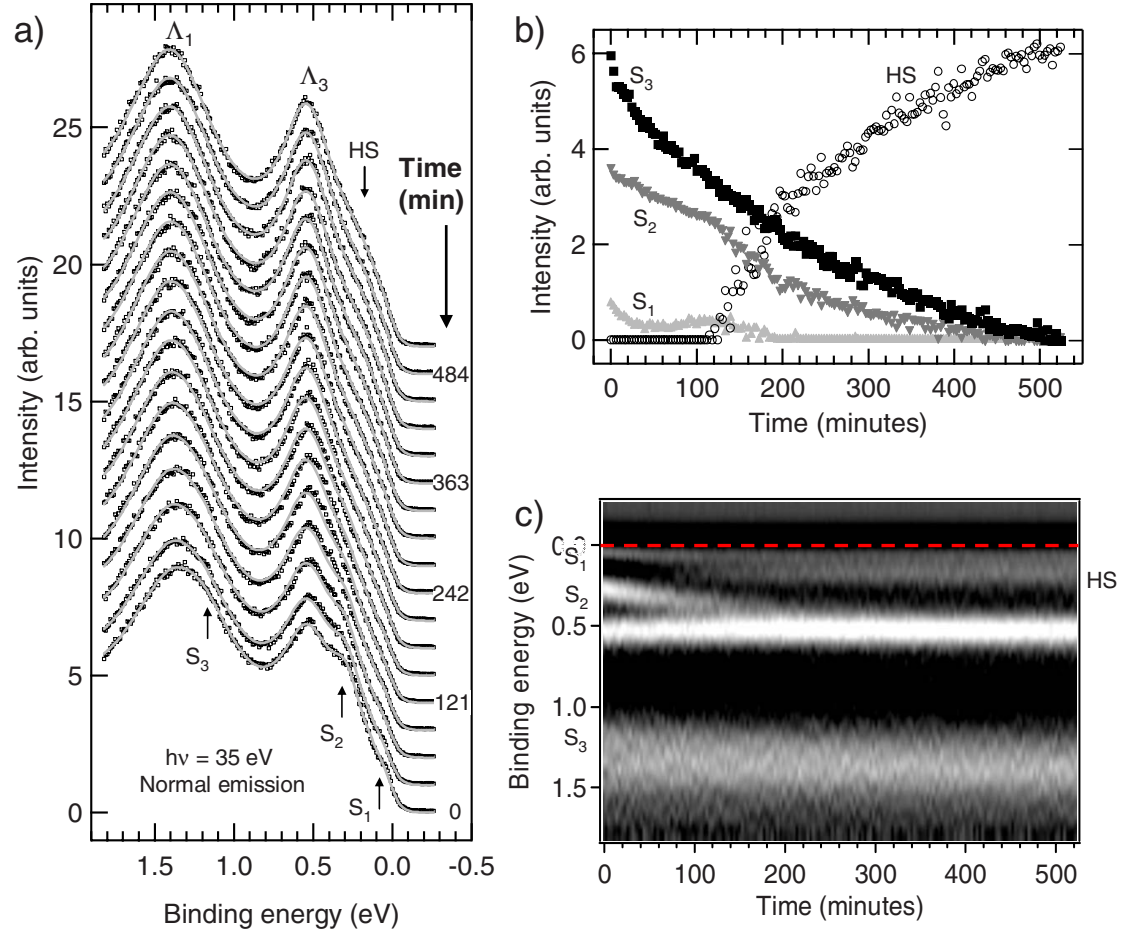

FIG. 5. (Color online) Room temperature normal emission spectra measured sequentially at $35 \mathrm{eV}$ as a function of residual gas exposure time. The existing surface states are indicated in the three panels $\left(S_{1}, S_{2}, S_{3}\right.$, and HS states). (a) shows some representative spectra (open squares) of the time evolution that the $\mathrm{Ni}(111)$ surface displays as it is progressively covered with hydrogen (the numbers on the right indicate the elapsed time). The superimposed gray curves correspond to the fits using six Lorentzian components and a linear background, multiplied with a Fermi-Dirac distribution. (b) represents the intensity variations of the surface state peaks (marked by the corresponding annotations) as a function of time (hydrogen exposure) as obtained from the fitting. (c) shows the second derivative of the smoothed raw spectra in gray scale (white represents high values) as a function of exposure time.

and a linear background, multiplied with a Fermi-Dirac distribution, was applied to these spectra. The resulting fits are shown as gray lines superimposed on the raw data. In this case, all parameters except for the peak intensities were kept constant during the fitting: the energy positions and the widths of both bulk $d$ bands and the hydrogen surface state were obtained from a previous fit to the last spectrum $(\mathrm{H}-$ passivated surface), while those of $S_{1}, S_{2}$, and the Tamm state peaks were obtained from the first spectrum (clean surface). Figure 5(b) shows the resulting intensity changes of the four Lorentzian peaks corresponding to the surface states. It can be seen that the three clean surface states ${ }^{45}$ decay in intensity at roughly the same rate, while the surface state related to the $\mathrm{H}$-passivated surface increases in intensity in the opposite way upon hydrogen adsorption.

The grayscale plot shown in Fig. 5(c) corresponds to the second derivative of the previously smoothed raw spectra as a function of the binding energy and the aging time. This data treatment allows us to enhance weak features ${ }^{46,47}$ and gives an independent way of checking the validity of the previously used fitting procedure. Again, it is observed that the $S_{1}$ state, ${ }^{48} S_{2}$ state, and the Tamm state $\left(S_{3}\right)$ peaks decay rapidly, while the hydrogen related surface state grows as the sample is exposed to the residual hydrogen gas of the vacuum chamber. As a minor difference, and since in this case the binding energy and width of the peaks are not kept fixed, we observe that the $S_{2}$ surface state moves slightly toward higher binding energies as the hydrogen coverage on the surface is increased, which is in agreement with the work of Himpsel et al. ${ }^{29}$ It is very likely that the same effect exists also for the $S_{1}$ state and the Tamm state, but due to the proximity to the Fermi edge of the former and the closeness to the $\Lambda_{1} d$ band of the latter, it is difficult to confirm this.

\section{CONCLUSIONS}

The surface states near normal emission of clean and H-passivated $\mathrm{Ni}(111)$ surfaces have been studied at RT by means of ARPES. It is found that, apart from the known $S_{1}$ and $S_{2}$ surface states (binding energies $\sim 0.02 \mathrm{eV}$ and $\sim 0.25 \mathrm{eV}$ ), a hidden surface state, $S_{3}$, is present with a binding energy of $\sim 1.19 \mathrm{eV}$. It is only observed by careful comparison of the spectral shape of the pristine and hydrogen saturated surfaces. The presence of this surface state is expected from the presented DFT calculations on $\mathrm{Ni}(111)$. It has a $d$-band origin and it is located in the $d$-band gap in between the $\Lambda_{3}$ and $\Lambda_{1}$ bulk bands, in close proximity to the latter.

Another surface state related to hydrogen adsorption has also been found, with a binding energy $\sim 0.22 \mathrm{eV}$. Its intensity increases with hydrogen coverage at the same rate as the other surface states of the clean $\mathrm{Ni}(111)$ surface are quenched. 


\section{ACKNOWLEDGMENTS}

We are indebted to M. Klöckner, C. Hess, and F. Dubi for their technical support. This work was performed at the Swiss Light Source, Paul Scherrer Institut, Villigen, Switzer- land. This work has been supported by the Swiss National Science Foundation, under Grant No. 200020-107783. T.O. was partly supported by Grant in Aid for Creative Basic Research from the Ministry of Education, Science, Sports and Culture of Japan.
*Present address: Department of Physics, University of Basel, Switzerland.

${ }^{1}$ M. C. Desjonquères and D. Spanjaard, Concepts in Surface Physics, 2nd ed., Springer Series in Surface Science (Springer, New York, 1998).

${ }^{2}$ H. Lüth, Surfaces and Interfaces of Solid Materials (SpringerVerlag, Berlin, 1995).

${ }^{3}$ S. G. Davison and M. Stęślicka, Basic Theory of Surface States, Monographs on the Physics and Chemistry of Materials Vol. 46 (Oxford University Press, New York, 1992).

${ }^{4}$ S. Hüfner, Photoelectron Spectroscopy, Springer Series in SolidState Sciences Vol. 82 (Springer-Verlag, Berlin, 1995).

${ }^{5}$ I. Tamm, Phys. Z. Sowjetunion 1, 733 (1932).

${ }^{6}$ W. Shockley, Phys. Rev. 56, 317 (1939).

${ }^{7}$ F. J. Himpsel and D. E. Eastman, Phys. Rev. Lett. 41, 507 (1978).

${ }^{8}$ G. Borstel, G. Thorner, M. Donath, V. Dose, and A. Goldmann, Solid State Commun. 55, 469 (1985).

${ }^{9}$ K. P. Kämper, W. Schmitt, and G. Güntherodt, Phys. Rev. B 42, 10696 (1990).

${ }^{10}$ M. Donath, F. Passek, and V. Dose, Phys. Rev. Lett. 70, 2802 (1993).

${ }^{11}$ J. Kutzner, R. Paucksch, C. Jabs, H. Zacharias, and J. Braun, Phys. Rev. B 56, 16003 (1997).

${ }^{12}$ W. Auwärter, Ph.D. thesis, Universität Zurich, 2003.

${ }^{13}$ T. Ohwaki, D. Wortmann, H. Ishida, S. Blügel, and K. Terakura, Phys. Rev. B 73, 235424 (2006).

${ }^{14}$ L. Magaud, A. Pasturel, P. Mallet, S. Pons, and J. Y. Veuillen, Europhys. Lett. 67, 90 (2003).

${ }^{15}$ J. Braun and M. Donath, Europhys. Lett. 59, 592 (2002).

${ }^{16}$ D. G. Dempsey, W. R. Grise, and L. Kleinman, Phys. Rev. B 18, 1550 (1978).

${ }^{17}$ F. J. Himpsel, J. A. Knapp, and D. E. Eastman, Phys. Rev. B 19, 2919 (1979).

${ }^{18}$ W. Eberhardt and E. W. Plummer, Phys. Rev. B 21, 3245 (1980).

${ }^{19}$ T. Greber, T. J. Kreutz, and J. Osterwalder, Phys. Rev. Lett. 79, 4465 (1997).

${ }^{20}$ T. J. Kreutz, T. Greber, P. Aebi, and J. Osterwalder, Phys. Rev. B 58, 1300 (1998).

${ }^{21}$ K. Giesen, F. Hage, F. J. Himpsel, H. J. Riess, and W. Steinmann, Phys. Rev. B 33, 5241 (1986).

${ }^{22}$ F. Passek and M. Donath, Phys. Rev. Lett. 69, 1101 (1992).

${ }^{23}$ S. Link, J. Sievers, H. A. Dürr, and W. Eberhardt, J. Electron Spectrosc. Relat. Phenom. 114-116, 351 (2001).

${ }^{24}$ M. Donath, Surf. Sci. Rep. 20, 251 (1993).

${ }^{25}$ E. W. Plummer and W. Eberhardt, Phys. Rev. B 20, 1444 (1979).

${ }^{26}$ S. G. Louie, P. Thiry, R. Pinchaux, Y. Pétroff, D. Chandesris, and J. Lecante, Phys. Rev. Lett. 44, 549 (1980).

${ }^{27}$ H. Yang and J. L. Whitten, J. Chem. Phys. 98, 5039 (1993).

${ }^{28}$ G. Kresse and J. Hafner, Surf. Sci. 459, 287 (2000).

${ }^{29}$ F. J. Himpsel, J. A. Knapp, and D. E. Eastman, Phys. Rev. B 19, 2872 (1979).

${ }^{30}$ W. Eberhardt, F. Greuter, and E. W. Plummer, Phys. Rev. Lett.
46, 1085 (1981).

${ }^{31}$ F. Greuter, I. Strathy, E. W. Plummer, and W. Eberhardt, Phys. Rev. B 33, 736 (1986)

${ }^{32}$ K. Christmann, O. Schoeber, G. Ertl, and M. Neumann, J. Chem. Phys. 60, 4528 (1974).

${ }^{33}$ M. Hoesch, T. Greber, V. N. Petrov, M. Muntwiler, M. Hengsberger, W. Auwärter, and J. Osterwalder, J. Electron Spectrosc. Relat. Phenom. 124, 264 (2002).

${ }^{34}$ E. Sjöstedt, L. Nordström, and D. J. Singh, Solid State Commun. 114, 15 (2000).

${ }^{35}$ G. K. H. Madsen, P. Blaha, K. Schwarz, E. Sjöstedt, and L. Nordström, Phys. Rev. B 64, 195134 (2001).

${ }^{36}$ P. Blaha, K. Schwarz, G. K. H. Madsen, D. Kvasnicka, and J. Luitz, WIEN2K, An augmented plane wave plus local orbitals program for calculating crystal properties, Vienna University of Technology, Vienna, 2001.

${ }^{37}$ K. Schwarz, P. Blaha, and G. K. H. Madsen, Comput. Phys. Commun. 147, 71 (2002).

${ }^{38}$ J. P. Perdew, K. Burke, and M. Ernzerhof, Phys. Rev. Lett. 77, 3865 (1996).

${ }^{39}$ P. E. Blöchl, O. Jepsen, and O. K. Andersen, Phys. Rev. B 49, 16223 (1994).

${ }^{40}$ G. Nicolay, F. Reinert, S. Hüfner, and P. Blaha, Phys. Rev. B 65 , 033407 (2001)

${ }^{41}$ F. Manghi, V. Bellini, J. Osterwalder, T. J. Kreutz, P. Aebi, and C. Arcangeli, Phys. Rev. B 59, R10409 (1999).

${ }^{42}$ All Lorentzian components displayed in Figs. 2 and 3 are represented multiplied with a Fermi-Dirac distribution.

${ }^{43}$ The decrease in intensity of $S_{1}$ when approaching the $\Gamma$ point, its proximity to the Fermi energy, and its weak intensity relative to the other components hinder its visualization for 30 and $32 \mathrm{eV}$, even though it is reproduced by this fitting procedure.

${ }^{44}$ Apparent changes in binding energy of $S_{2}$ are related to normal experimental errors, such as angular misalignment with respect to normal emission, Fermi energy determination, or beamline energy resolution limitations.

${ }^{45}$ Fitting the $S_{1}$ state is somewhat problematic due to its proximity to the Fermi energy and to its small intensity when compared to the other states. Nevertheless, its trend follows the behavior of the other clean $\mathrm{Ni}(111)$ surface states.

${ }^{46}$ A. Mugarza and J. E. Ortega, J. Phys.: Condens. Matter 15, S3281 (2003).

${ }^{47}$ J. Lobo, E. G. Michel, A. R. Bachmann, S. Speller, J. Kuntze, and J. E. Ortega, Phys. Rev. Lett. 93, 137602 (2004).

${ }^{48}$ The second derivative method enhances slope changes, thus marking the position of the peaks. The leading edge in this kind of data treatment is always observed in the second derivative, unless the Fermi step is previously removed from the data. This data manipulation has not been performed, so we obtain some intensity mixing between the Fermi edge and the $S_{1}$ surface state peak. 